\title{
Review of: "Modulation of circadian clock by crude drug extracts used in Japanese Kampo medicine"
}

Jie Liu

Potential competing interests: The author(s) declared that no potential competing interests exist.

This is an interesting study that examined the modulation effects of circadian clock by 137 crude drug extracts used in Japanese Kampo medicine. This work is a logical extension of their recent publications (PMID 29666146) using human U2OS cells stably expressing the clock reporter Bmal1-dLuc to identify circadian modulators from existing drugs. The period change and phase change of 17 hit crude drug extracts and 8 major ingredients of hit crude drugs are nicely presented in the U2OS cells, an innovative approach in circadian research. Effects of three crude drug extracts were further examined using Rat-1 fibroblasts expressing Per2-luc and lung explants from Per2::luciferase knockin mice, and the effects of Allii Chinese Bulbus extract are further examined in zebrafish larvae. Overall, the experiment systems are innovative measures allowing the study of crude drug extracts from traditional medicines. 